\title{
Tonal priming is resistant to changes in pitch height
}

\author{
Jon B. Prince ${ }^{1}$ • Dominique T. Vuvan ${ }^{2}$ • Mark A. Schmuckler ${ }^{3}$ - Thomas T. Scott-Clark ${ }^{1}$
}

Published online: 25 April 2015

(C) The Psychonomic Society, Inc. 2015

\begin{abstract}
Research on tonal priming has consistently shown that tonally expected events are processed more efficiently and has confirmed that the locus of the effect is cognitive rather than sensory. However, it is also important to investigate the role of pitch height, because models of tonal priming collapse across octaves, yet it is possible that pitch height may modulate the effectiveness of tonal priming. We systematically tested this issue by varying the pitch heights of a related (tonic) or a less-related (subdominant) target chord following a tonal context. Musically untrained participants $(N=30)$ made speeded consonant/dissonant judgments of the final chord of an eight-chord sequence. The effects of tonal priming emerged in accuracy and reaction time measures for all octaves, except for a ceiling effect on accuracy in the matching (original pitch height) condition. In a second experiment, we increased the shift to two octaves and compressed the chords to eliminate overlap between the target and context chords; again, tonal priming emerged. These findings have implications for the behavioral study of tonal priming and support the assumption of octave equivalence in computational models.
\end{abstract}

Keywords Music cognition $\cdot$ Pitch perception $\cdot$ Priming

Jon B. Prince

j.prince@murdoch.edu.au

1 Department of Psychology and Exercise Science, Murdoch University, Perth, Western Australia

2 Department of Psychology and International Laboratory for Brain, Music, and Sound Research, Université de Montréal, Montreal, Québec, Canada

3 University of Toronto Scarborough, Toronto, Ontario, Canada
Cognitive processes are fundamentally based on prediction: By predicting the most likely subsequent event, an organism has a better chance of reacting to it in a beneficial way. Some of these expectancies emerge from relatively simple surface information, but predictions are also formed for events derived from deeper structural patterns. For example, an increase in the amplitude of successive sound events creates an expectation for the next sound to be yet louder. This prediction arises from information immediately available at the surface level of the stimulus. Conversely, a semantic word prime need not have any acoustic similarity to its target (e.g., doctor-nurse). Thus, both sensory and cognitive priming contribute to the formation of expectancies.

The roles of sensory and cognitive priming are especially important in music, in particular with regard to tonal priming (for a review, see Collins, Tillmann, Barrett, Delbé, \& Janata, 2014). This phenomenon typically refers to the fact that for listeners enculturated in Western music, a prime chord (multiple simultaneous notes) creates a strong expectation for a musically related chord (Bharucha \& Stoeckig, 1986; Bigand, Poulin, Tillmann, Madurell, \& D'Adamo, 2003). This concept is equally applicable to monophonic sequences of single notes, or melodies (Boltz, 1989; Marmel \& Tillmann, 2009; Marmel, Tillmann, \& Delbe, 2010).

In any given musical key, the tonic chord is the most musically expected, psychologically stable, and frequently occurring chord (Krumhansl, 1990). It consists of the most expected/stable/frequent pitch classes in that key. By virtue of this privileged status, listeners are faster and better at processing events that occur synchronously with a tonic chord, regardless of their level of formal musical training (Bigand \& Poulin-Charronnat, 2006). These need not be musical tasks - tonal priming is evident in unrelated tasks such as phoneme identification (Bigand, Tillmann, Poulin, D'Adamo, \& Madurell, 2001), and even visual object recognition (Escoffier \& Tillmann, 2008). Such 
findings suggest that tonal priming modulates central attentional mechanisms rather than music-specific ones. This effect consists of both facilitation of related chords and inhibition of lessrelated chords (Tillmann, Janata, Birk, \& Bharucha, 2003, 2008), rather than a simple graded inhibition whereby the tonic chord interferes the least.

Before accepting that tonal priming is a cognitive phenomenon, explanations based on the raw sensory information must be ruled out. The hierarchy of chords in musical stability is correlated with their acoustic similarity, so perhaps sensorybased repetition priming may drive the effect rather than a cognitive musical relation. However, tonal priming persists after removing the overlapping frequency spectra of musically related chords, undermining a sensory explanation (Bharucha \& Stoeckig, 1987). Additionally, when sensory (repetition priming) and cognitive (tonal priming) are placed in direct conflict, the cognitive prime prevails (Bigand et al., 2003). Bigand et al. (2003) defined sensory priming as the number of pitch classes shared by the target and the preceding musical context (i.e., how often each pitch class occurs). Yet, even when the context shared more tones with the less musically related subdominant chord, the tonic chord was primed the most. Finally, violating the good continuation of voiceleading (the principle of favoring small pitch changes between subsequent notes and filling in larger leaps; see Meyer, 1973; Narmour, 1990) slows reaction times to targets, but it does not influence the effect of tonal priming (Poulin-Charronnat, Bigand, \& Madurell, 2005).

Nevertheless, sensory priming is still relevant to musical processing; chord repetition can actually decrease the effectiveness of tonal priming (Bigand, Tillmann, Poulin-Charronnat, \& Manderlier, 2005). Furthermore, at very short delays of $50 \mathrm{~ms}$ (half the minimum delay between musical notes; see Huron, 2001), acoustic similarity overpowers musical priming, but by $500 \mathrm{~ms}$, the cognitive component is the primary determinant of priming (Tekman \& Bharucha, 1998). Leman (2000) proposed a model to account for tonality by using short-term memory and periodicity pitch, implying that tonality is in fact a sensory phenomenon and need not involve any cognitive processing. However, more recent evidence has argued strongly against a purely sensory account of tonal priming (Bigand et al., 2003; Marmel et al., 2010; Vuvan \& Schmuckler, 2011). A recent comprehensive review, meta-analysis, and updated modeling of numerous priming studies demonstrated that both sensory and cognitive factors play roles in tonal priming, and moreover, that cognitive factors prevail as the more influential ones (Collins et al., 2014). These authors predicted the strength of priming effects using three factors - periodicity pitch (sensory), the distribution of pitch classes (chroma vector), and the relative activation of musical keys (tonal space). The second and third factors are both cognitive, in that they represent derived information not immediately available from the stimulus surface. The cognitive factor of tonal space was the most influential one, and the addition of a hybrid hypothesized/empirical probability of closure further improved their modeling of priming effects. Other recent simulation work has highlighted the role of auditory short-term memory in tonal priming as a way of distinguishing musical and linguistic syntax processing (Bigand, Delbé, Poulin-Charronnat, Leman, \& Tillmann, 2014).

Despite their attention to the potential contributions of sensory information to music perception, models of tonal priming have neglected the role of pitch height. The predominant model, MUSACT (Bharucha, 1987), assumes 12 pitch nodes that correspond to the number of unique pitch classes used in Western music (per octave). Because this model collapses across octaves, it cannot address the role of pitch height variation in tonal priming. Without a component of pitch height in MUSACT, a primed chord should receive the same benefit regardless of the octave in which it occurred. Although this model is successful at modeling perceptual data (see Collins et al., 2014), including a self-organizing neural network instantiation (Tillmann, Bharucha, \& Bigand, 2000), this prediction has not been tested empirically. Because the model proposed by Collins et al. (2014) uses an auditory input as a signal, it should be capable of addressing the role of pitch height. However, despite data based on the model fitting a majority of the prior studies well, no specific testing of the effect of pitch height on tonal priming has yet been conducted.

On the one hand, there are reasons not to expect that changes in pitch height would vitiate tonal priming. Octave equivalence is the concept that pitches with the same class (e.g., C) that are played in different octaves are musically equivalent, and this is essentially universal across musical cultures (Brown \& Jordania, 2013). Experimental dissociations between pitch class and pitch height (Kallman \& Massaro, 1979; Shepard, 1982) support octave equivalence, which emerges in 3-month-old infants (Demany \& Armand, 1984), and even rhesus monkeys (Wright, Rivera, Hulse, Shyan, \& Neiworth, 2000). Moreover, the pitches of a melodic sequence are encoded according to their tonal function rather than their physical properties. For example, detecting an alteration to a pitch was hardest when it changed to a musically related pitch, even though this was also the largest physical change of frequency (Trainor \& Trehub, 1994). Because tonal priming depends on encoding the tonal function of musical events, it is possible that changes in pitch height would not influence its effectiveness.

On the other hand, pitch height is not a trivial characteristic of music, as has been evidenced by its indispensable roles in stream segregation (Bregman, 1990; Dowling, 1973; van Noorden, 1975) and melodic recognition (Deutsch, 2013; Dowling, 1978). Recognizing a familiar melody is extremely difficult if the notes are dispersed randomly across different octaves, while retaining their pitch class (Deutsch, 1972; Dowling, 1984; Dowling \& Hollombe, 1977). More 
generally, large changes in pitch height between subsequent melodic notes increase the difficulty of apprehending the tonal structure of a melody (Bharucha, 1984; Cuddy, Cohen, \& Mewhort, 1981), which typically use small steps between notes. Whether pitch height also affects tonal priming is an open question; pitch height can influence the functionality of the tonal hierarchy, which operates well only within the octaves typically used in Western music (Russo, Cuddy, Galembo, \& Thompson, 2007). Russo et al. used the standard probe-tone procedure of playing a musical sequence that establishes a musical key, followed by a probe tone at one of 12 pitch classes, which listeners rate for their goodness of fit with the preceding context (Krumhansl \& Kessler, 1982). Russo et al. extended this procedure by testing pitches between $\mathrm{E}_{0}^{\mathrm{b}}$ and $\mathrm{C}_{8}(20-4186 \mathrm{~Hz})$, and found that the standard tonal hierarchy profile is most obvious within the range of $A_{1}$ to $A_{5}$ $(55-880 \mathrm{~Hz})$. Beyond this range, it is increasingly difficult to recover the tonal hierarchy, to the point where it breaks down altogether at the most extreme ranges (the highest and lowest octaves on the piano).

The goal of the present research was to assess the validity of the untested assumption of octave equivalence in tonal priming. In other words, does tonal priming still work when the target chord occurs in a different octave? We used a standard paradigm in which a sequence of six chords establishes a musical key (not including the tonic chord), and ends with either the most expected tonic chord or the less expected (yet consonant) subdominant chord. Participants provided speeded judgments of the final (target) chord, which should be facilitated for tonic chords over subdominant chords. The critical manipulation that we added was to vary the pitch height of the target chord. There were three potential outcomes - the pitch height manipulation could have no effect (tonic better than subdominant, regardless of octave), or could weaken tonal priming (tonic still better, but less so at different octaves), or could abolish tonal priming altogether (no advantage of the tonic over the subdominant in other octaves).

\section{Experiment 1}

\section{Method}

Participants A group of 30 participants took part in Experiment 1, recruited from the undergraduate community at Murdoch University and run individually. The participants were reimbursed with course credit. Their average age was $26.7(S D=10.8)$, and they had had an average of 3.3 years of formal musical training $(S D=4.0)$.

Apparatus Custom MATLAB scripts (The Mathworks, 2004) using the Psychophysics Toolbox (Brainard, 1997) were used to create the experimental interface on a PC running Windows 7. The stimuli were composed in Finale SongWriter 2010 using an acoustic piano timbre and exported to .wav files. Participants were seated in a quiet room and listened to the stimuli over Sennheiser HD280Pro headphones.

Stimuli Twelve unique sequences of six chords (the chord context) were prepared that clearly established the musical key of $\mathrm{C}$ major (see Fig. 1 for an example). The average maximum key correlation (Krumhansl, 1990; Krumhansl \& Schmuckler, 1986) was .82 $(S D=.05)$, and the duration of each chord was $500 \mathrm{~ms}$. The tonic chord (C major) did not occur in the chord context, but any other diatonic triad was allowed. This arrangement was equivalent to the "subdominant in context" condition of Bigand et al. (2003), in which the less-related subdominant triad could occur in the context, but not the tonic.

Two additional chords followed each chord context, composed of either a dominant and a tonic chord (V-I cadence) or a tonic and a subdominant chord (I-IV cadence). The tonic chord is the most musically expected, whereas the subdominant is less expected (but still legal). In terms of cognitive and sensory priming, the tonic chord receives the strongest cognitive priming, whereas sensory priming is strongest for the subdominant chord, because it was heard earlier in the sequence. Thus, harmonic relatedness was manipulated by varying which chord pair occurred after the context. Importantly, both cadence types (V-I and I-IV) are identical transitions when presented outside of a preceding musical context. For example, in the key of $\mathrm{C}$ major, a $\mathrm{V}-\mathrm{I}$ cadence consists of a $\mathrm{G}$ major chord followed by a $\mathrm{C}$ major chord; however, in the key of $\mathrm{G}$ major the exact same chords define a I-IV cadence. Thus, the manipulation of harmonic relatedness was not confounded with the identity of the final (target) chord. Any difference between harmonic relatedness conditions could therefore only be a result of the preceding six-chord context that primed the tonic over the subdominant chord (via cognitive priming) or the reverse (via sensory priming).

The participants' task was to judge whether the final chord was consonant or dissonant. Adding an additional note to a chord that does not belong in the key of $\mathrm{C}$ major makes it sound dissonant (i.e., subjectively rough, unpleasing, or clashing). Consonant chords had no added notes, and therefore sounded smooth, pleasing, or not clashing. For half of the dissonant trials, the added note was one semitone above the root of the chord (\#1), and for the other half it was one semitone above the fifth, or top note of the triad (\#5). The added note was always within the pitch range of the consonant target chord, as shown in Fig. 1. Each participant heard equal numbers of consonant and dissonant trials. 


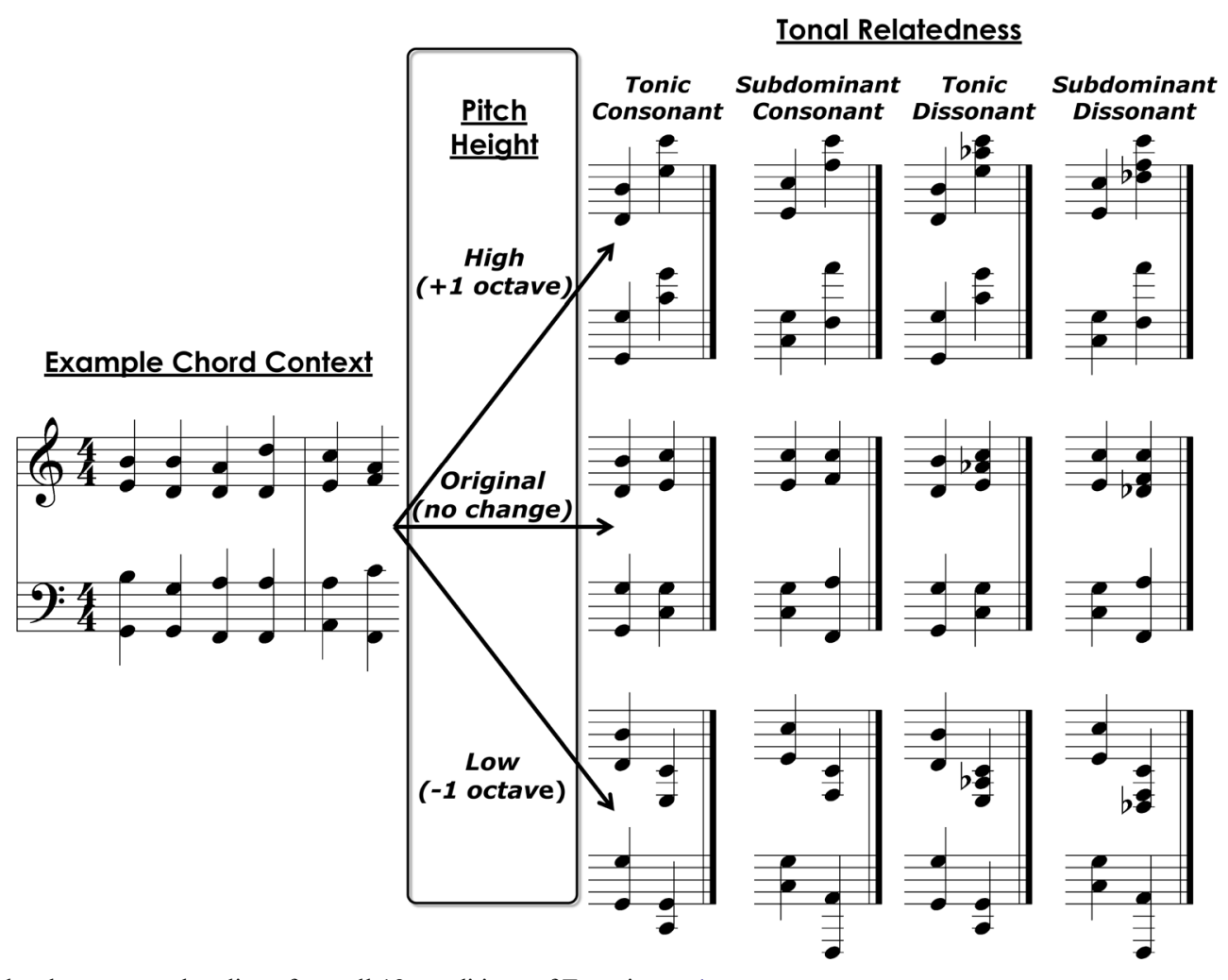

Fig. 1 Example chord context and endings from all 12 conditions of Experiment 1

The manipulation of primary interest was that of the target's pitch height. The final chord (target) was presented either in its original pitch register, shifted up one octave, or shifted down one octave (see Fig. 1). Crossing the 12 sequences with the two levels of harmonic relatedness, two levels of consonance, and three levels of pitch height resulted in a total of 144 conditions. Participants heard each condition twice, giving 288 trials. There were three versions of each sequence, transposed to either the key of C, F, or G major. Assignment of the sequences to keys was counterbalanced, such that each participant heard equal numbers of sequences in each key.

Procedure Participants first provided informed consent and completed a background questionnaire on their musical experience. Subsequently, they were seated in the experimental room and received instructions on the task, which was to indicate whether the final chord of a sequence was consonant or dissonant. The experimenter explained the concepts of consonance and dissonance, and participants did 24 practice trials in which a single chord was presented, which was either consonant or dissonant (as defined above). The experimenter was present throughout the practice trials in order to answer questions. Once the participants understood the task and had completed the practice trials, they continued to the full experiment (288 trials, mixed design). The experimenter debriefed the participants once they had completed the experiment, which took approximately $30 \mathrm{~min}$.

\section{Results}

Participants performed well at the task, with an average proportion correct of $.85(S D=.09)$. Valid reaction times (from correct trials only) averaged $484 \mathrm{~ms}(S D=18)$. Accuracy and reaction time correlated negatively $(r=-.32)$, ruling out a speed-accuracy trade-off. All subsequent analyses included the consonant trials only, since the added out-of-key notes in dissonant trials defeated the manipulation of harmonic relatedness. A 2 (Harmonic Relatedness: tonic or subdominant) $\times$ 3 (Target Pitch Height: high, original, or low) repeated measures analysis of variance (ANOVA) on the accuracy data revealed significant main effects for both factors and a significant interaction; these effects appear in Fig. 2. The main effect of harmonic relatedness was that participants were more accurate on tonic than on subdominant trials, $F(1,29)=28.1, p=$ $.003, \eta^{2}=.06 .^{1}$ The main effect of pitch height emerged from better performance in the original pitch height condition than in the high and low conditions, $F(2,58)=15.9, p<.001, \eta^{2}=$ .26 (the high and low conditions differed by only .026 , pairwise comparison $p=1$ ). The interaction, $F(2,58)=6.3$, $p<.001, \eta^{2}=.03$, revealed that there was no effect of harmonic relatedness on accuracy in the original pitch height condition, $t(29)=0.53, p=.6$, whereas such an effect did

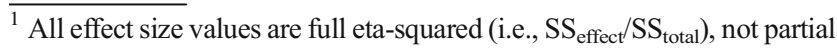
eta-squared.
} 


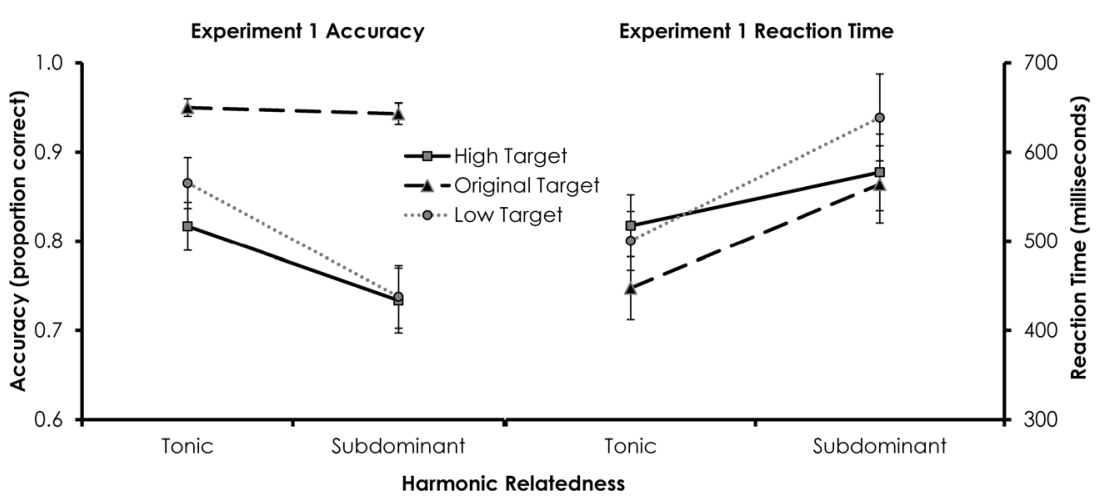

Fig. 2 Accuracy and reaction time data from Experiment 1. Error bars are standard errors of the means

emerge in both the high condition, $t(29)=2.93, p=.007$, and the low condition, $t(29)=4.62, p<.001$.

The same ANOVA on the reaction time data also showed main effects of harmonic relatedness, $F(1,29)=39.6, p<$ $.001, \eta^{2}=.19$, and pitch height, $F(2,58)=3.3, p=.045, \eta^{2}$ $=.05$; these data also appear in Fig. 2 . As with the accuracy data, participants did better for tonic trials and for the original pitch height condition, although the only significant pairwise difference was between the low and original pitch height conditions, mean difference $=64 \mathrm{~ms}, p=.04$. We again observed an interaction, $F(2,58)=3.2, p=.049, \eta^{2}=.02$, in this case because the effect of harmonic relatedness was smaller in the high pitch height condition, although the tonic and subdominant conditions were significantly different at all pitch heights: $t(29)=2.78, p=.01$ (high), $t(29)=5.35, p<.001$ (original), and $t(29)=4.57, p<.001$ (low).

Expertise analysis To determine whether musical expertise influenced the effectiveness of tonal priming or its immunity to pitch height changes, years of formal training was correlated with the difference scores (between the tonic and subdominant relatedness conditions) for accuracy and reaction times. No significant correlations were apparent, indicating that musical training did not influence the strength of tonal priming in any pitch height condition (highest $r=.24, p=.20$ ).

\section{Discussion}

Changing the pitch height of a sequence-final target chord impaired the overall accuracy and reaction time on a speeded consonant/dissonant judgment, similar to the effect of violating voice-leading (Poulin-Charronnat et al., 2005). Manipulating the harmonic relatedness also influenced performance, with participants performing better for targets that were more musically expected, regardless of musical experience. Thus, cognitive priming of musical expectations overrode the effects of sensory (repetition) priming (Bigand et al., 2003) and was not dependent on formal musical training (Bigand \& PoulinCharronnat, 2006). Most critically, the effect of harmonic relatedness emerged at all target pitch height conditions (in accuracy and/or reaction time), demonstrating that tonal priming is not limited to the pitch range of the preceding context. These findings confirm and extend existing tonal priming effects in the literature, which heretofore have assumed octave equivalence. These data are the first to address this assumption directly, and argue in the affirmative for tonal priming being impervious to pitch height manipulations.

The accuracy data of the original pitch height condition showed no effect of tonal priming, likely due to a ceiling effect (accuracy was 95\%, and error bars were smallest in this pitch height condition). However, the reaction time measure showed strong tonal priming. Thus, maintaining the same pitch height for the target made the task sufficiently easy that manipulating harmonic relatedness had no observable effect on the accuracy measure, instead affecting reaction times.

The effect of tonal priming on reaction times was significant in all pitch height conditions but was nominally smallest in the high pitch height condition, suggesting that tonal priming could diminish further at larger pitch height changes. However, the low pitch height condition argues for the robustness of tonal priming across pitch heights, since we observed no reduction in the strength of tonal priming whatsoever (as compared to the original condition with no pitch height change). Yet here, too, there may be a problem, because participants noted that targets in the low pitch height condition sounded "muddy," or more dissonant, than targets in the original or high pitch height conditions. It is unclear how this phenomenon might affect tonal priming, and moreover, whether the absolute pitch ranges of the context and target are important.

These findings therefore warrant further investigation of how pitch height might influence tonal priming. That is, what are the roles of the size of the pitch height change and the absolute pitch range in determining whether tonal priming is functional? Experiment 2 was designed to answer these questions.

\section{Experiment 2}

Although Experiment 1 strongly suggested that pitch height has a negligible (if any) impact on tonal priming, the findings raised 
further questions. In Experiment 1, the one-octave change did not eliminate the pitch range overlap; for instance, the two lowest notes of the target chord matched the pitch range of the two upper notes of the penultimate chord (for the high conditionthe reverse was true for the low condition). Thus, despite the change of pitch register, the remaining overlap may have fostered tonal priming effects. In Experiment 2, the shift was increased to two octaves in order to test whether tonal priming remains when the pitch height change is dramatically larger. For the downward shift condition, the chord context was transposed up two octaves and the target remained in the original (untransposed) position, to eliminate the muddy dissonant sound of the lowest chords in Experiment 1. Observing tonal priming effects in these conditions would provide strong validation of the octave equivalence assumption in tonal priming models.

\section{Method}

Participants A new set of 30 participants from the same pool were recruited for Experiment 2, with an average age of 26.6 years $(S D=9.5)$ and 3.8 years of formal musical training, on average $(S D=4.7)$.

Apparatus All of the apparatus was the same as in Experiment 1.

Stimuli The three pitch height conditions were as follows: target up two octaves, target at the original pitch height, and target down two octaves. For the last condition, the context was shifted up two octaves, and the pitch height of the target remained unchanged (thus, two octaves lower than the expected pitch height).

The chord sequences were compacted into "block" chords that spanned less than an octave, which was a considerably smaller range than the chords of Experiment 1 (see Fig. 3). This compression maximized the gap between the context and target, in order to prevent pitch height overlap from influencing the data. In fact, the empty gap between the last two chords in the pitch change conditions (e.g., for the high condition, the distance between the highest note of the penultimate chord to the lowest note of the target) averaged 17 semitones $(S D=6.0)$.

The design was the same as in Experiment 1, with 12 sequences, two levels of harmonic relatedness, two levels of consonance, and three levels of pitch height. Again all 144 conditions had two repetitions, resulting in 288 trials overall.

Procedure The procedure was identical to that of Experiment 1.

\section{Results}

The average proportion correct was $.88(S D=.08)$, and the average reaction time of valid trials was $487 \mathrm{~ms}(S D=22)$. We found no evidence of a speed-accuracy trade-off, since accuracy and reaction time were negatively correlated, $r=$ - .19. Again, only consonant trials were analyzed. A 2 (Harmonic Relatedness) $\times 3$ (Pitch Height) repeated measures ANOVA of the accuracy data recovered a main effect of relatedness, $F(1,29)=11.4, p=.002, \eta^{2}=.06$, because accuracy was higher for the tonic than for the subdominant (as in Exp. 1; see Fig. 4). There was also a main effect of pitch height, $F(2$, $58)=19.6, p<.001, \eta^{2}=.29$, due to the best performance occurring for the original pitch height targets, intermediate performance for the high targets, and the worst performance for the low targets (all pairwise comparisons significant, $p<.05$ ). We observed no interaction between relatedness and pitch height, $F(2,58)<1$, n.s.

Repeating the same analysis with the valid reaction time data revealed a main effect of relatedness, $F(1,29)=7.8, p=.009, \eta^{2}$ $=.05$ (faster for tonic than for subdominant targets). We also found an effect of pitch height, $F(2,58)=3.9, p=.03, \eta^{2}=.07$, because participants were faster for high than for low targets, mean difference $=53 \mathrm{~ms}, p=.048$ (original targets differed from neither high nor low, $p \mathrm{~s}=.38$ and .49 , respectively). There was no interaction, $F(2,58)=1.4, p=.25, \eta^{2}=.01$.

Expertise analysis The analysis correlating accuracy and reaction time difference scores between tonic and subdominant relatedness conditions with years of formal training (as in Exp. 1) showed no significant relationships between the variables (highest $r=.28, p=.14$ ). Therefore, there was no connection between the strength of tonal priming and musical expertise.

\section{Discussion}

In Experiment 1, the wide chord voicings and smaller octave shift meant that there was always some overlap between the penultimate chord and the target, which may have aided the perseveration of tonal priming. Compressing the chords and doubling the octave shift in Experiment 2 eliminated any remaining overlap in the high and low conditions, yet tonal priming was still operational, regardless of the target's pitch height. Furthermore, and unlike Experiment 1, the effects of tonal priming did not differ across pitch height conditions. In other words, tonal priming persisted despite extreme changes in pitch height. Again, we found no effects of musical expertise, reinforcing the ubiquitous nature of tonal priming across Western listeners, regardless of training. Together, these findings strongly endorse tonal priming as being immune to large variations in pitch height.

The main effect of relatedness on reaction times was smaller in Experiment 2. However, the critical question was whether the effect of relatedness would change across pitch height conditions, not across experiments. Accordingly, the lack of an interaction between these factors (in both experiments) is the most important evidence of the resilience of tonal priming across pitch heights. 


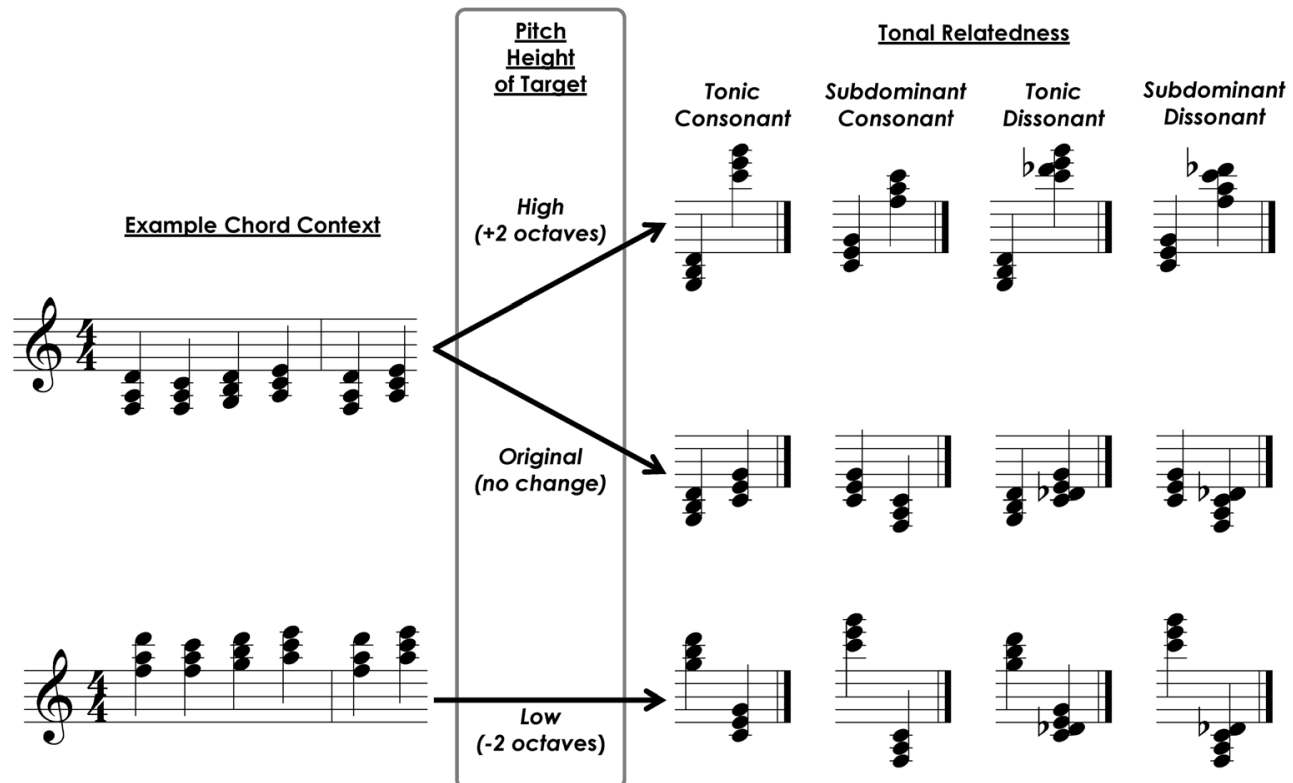

Fig. 3 Example chord context and endings from all conditions of Experiment 2

Given that changing the pitch height of the target decreased participants' accuracy, it was reasonable to predict that larger changes would result in greater performance penalties. It is therefore surprising that the advantage of the original condition was actually smaller in Experiment 2-the lines representing the low and high condition in Fig. 4 are closer to the original condition than they are in Fig. 2 (Exp. 1). To be clear, shifting the pitch height of the target made consonance/dissonance judgments more difficult, but it caused no attenuation in tonal priming. Indeed, the harmonic relatedness effect was nominally (but not significantly) strongest in the target-up condition, entirely inconsistent with the notion that tonal priming would decrease when the context and target were at different pitch heights.

On the whole, these data provide no reason to expect that further increases in pitch height would be detrimental to tonal priming. Moreover, such large shifts are virtually nonexistent in standard Western music, and anything beyond two octaves would be musically untenable (indeed, even these shifts are not particularly musical).

\section{General discussion}

Two experiments were conducted to investigate how pitch height differences between context and target chords might affect tonal priming. In Experiment 1, we used a conventional paradigm to test whether shifting the target chord up or down an octave would influence the effectiveness of tonal priming. Although pitch height changes impaired performance overall, the effects of tonal priming remained in both the accuracy and reaction time measures. In Experiment 2, we used a larger shift ( \pm 2 octaves) of the target to eliminate pitch register overlap with the context, and tonal priming still occurred in these cases. These data strongly

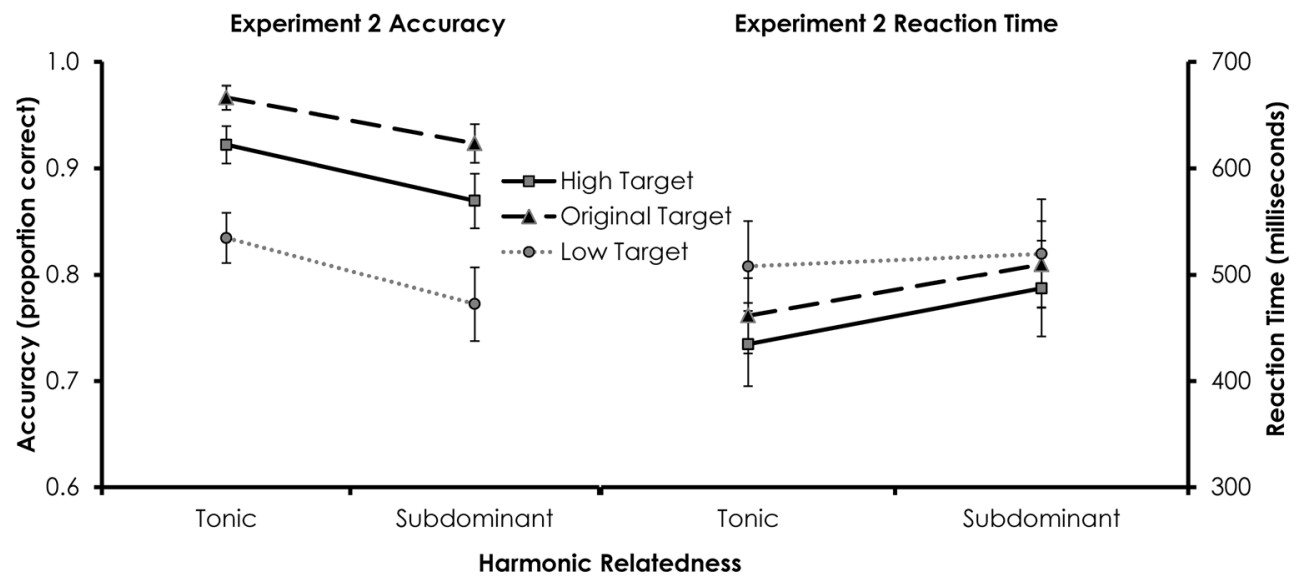

Fig. 4 Accuracy and reaction time data from Experiment 2. Error bars are standard errors of the means 
endorse the consistency of tonal priming in the face of pitch height manipulations.

The primary motivation of this research was to test whether the assumption of octave equivalence in the tonal priming literature is valid. This issue is particularly relevant to computational models of tonal expectancies that assume octave equivalence, in particular MUSACT (Bharucha, 1987). Indeed, in its current form this model cannot predict any effect of pitch height whatsoever, although given the present findings, it would be possible to extend MUSACT by adding an independent modular component of pitch height. Of course, the more recent model of Collins et al. (2014) automatically includes pitch height, via the periodicity pitch component. Despite the near-universality and early developmental trajectory of octave equivalence, however, there has been some controversy regarding the existence of octave equivalence in psychophysical research (for a discussion, see Burns, 1999), and some evidence that musical training develops the phenomenon (Krumhansl \& Shepard, 1979). Nevertheless, more recent research has shown that failures to observe octave equivalence are most likely to be due to methodological difficulties, and that the phenomenon is not dependent on training (Hoeschele, Weisman, \& Sturdy, 2012). The present findings concur with this perspective - although moving the target to a different octave decreased performance overall, the tonal functions were equivalent regardless.

Although this research discounts the effect of pitch height on tonal priming, it is unquestionable that pitch height is a fundamental component of music perception, affecting the very identity of a musical excerpt, indicating emotional cues (Huron, Kinney, \& Precoda, 2006; Juslin \& Laukka, 2003), and influencing stream segregation (Bregman, 1990; Dowling, 1973). Indeed, the sequence of changes in pitch height, otherwise known as melodic contour, is a primary component in the perception of musical pitch (Deutsch, 2013; Dowling, 1978; Schmuckler, 2004, 2009). Additionally, the most comprehensive analysis of the tonal priming literature argues for a role (albeit subsidiary) of the sensory characteristic of periodicity pitch (Collins et al., 2014).

The independence of pitch height and tonal priming was not a foregone conclusion. Initial research into tonality showed that pitch height can influence the relative stability of different pitch classes (Krumhansl, 1979; Krumhansl \& Shepard, 1979). Specifically, for some listeners, probe pitches closer in pitch height to the preceding context received higher goodness-of-fit ratings. Subsequent work used circular tones (Shepard tones) that obscured any notion of pitch height (Shepard, 1964), enabling studying tonality independently of pitch height effects (Krumhansl \& Kessler, 1982). Other behavioral research showed that introducing more complex contours reduces the ability to extract the underlying harmonic structure of a melody (Cuddy et al., 1981), and that anchoring out-of-key tones to in-key tones depended critically on their pitch height proximity (Bharucha, 1984). Additionally, the variable strength of the tonal hierarchy in octaves outside the range of typical music shows that tonality is not impervious to changes in pitch height (Russo et al., 2007). The vast majority (97\%) of the pitches tested in these experiments were within the octave range in which Russo et al. successfully recovered the tonal hierarchy (Octaves 1-6), so it is perhaps to be expected that tonality (and thus, tonal priming) was fully operational. It is nonetheless surprising that we observed no decrease in tonal priming at the extreme octaves, as would be predicted by a decreased role of tonality as pitches strayed beyond the typical bounds of music (Octaves 2-5).

More generally, introducing drastic changes in pitch height is not simply a sensory change - it violates the voice-leading principles of music. Again, previous research has shown that violating voice-leading makes processing musical chords more challenging, but has no effect on tonal priming (PoulinCharronnat et al., 2005). In the present study, our manipulation of pitch height necessarily also changed voice-leading, and thus our findings show that tonal priming is immune to concomitant changes to voice-leading and pitch height. Like voice-leading, preserving normal pitch height is also a learned schematic expectation, and the present data showed an overall cost to changing the pitch height. But the other schematic expectation of tonal relatedness remained intact regardless of the change in pitch height, dissociating these two types of schematic expectation. In other words, pitch height and tonal relatedness are not general features that contribute to an overall sense of schematic expectation, but are demonstrably independent processes in the perception of music. This perspective is reinforced by the successful regression modeling of tonal priming using independent predictors (Collins et al., 2014).

Neuroimaging research has substantiated the independence of pitch height and pitch class, because changing them activated distinct areas of auditory cortex (Warren, Uppenkamp, Patterson, \& Griffiths, 2003). Furthermore, although areas of rostromedial frontal cortex have shown specific activation patterns to different musical keys, the exact mapping is flexiblethe topography of which key aligned to which activation pattern varied across scanning sessions (Janata et al., 2002). Thus, brain activity in response to manipulations of tonality seems unaffected by the exact pitch height. With specific regard to tonal priming (using the same speeded consonant/ dissonant judgment task), the inferior frontal cortex responds differently to related and unrelated chords (Tillmann, Janata, \& Bharucha, 2003).

Each chord used in these experiments consisted of several pitches that extended over an interval of at least seven semitones (see Fig. 3), and sometimes nearly three octaves (see Fig. 1). It is uncertain whether tonal priming remains resistant to pitch height changes in a context with a much smaller pitch range, such as a monophonic melody, which consists of single notes. Tonal priming is functional in a melodic context (Marmel, Perrin, \& Tillmann, 2011; Marmel \& Tillmann, 2009; Marmel et al., 2010; Marmel, Tillmann, \& Dowling, 
2008), but melodies rarely exceed a pitch range of 1.5 octaves (typically the range is much smaller). Future research should focus on testing the effect of pitch height on tonal priming in a melodic context. These data argue against a role of pitch height, but assumptions are always worth testing, and replications are particularly valuable in the current scientific context (Pashler \& Wagenmakers, 2012).

The primary message from this research is that tonal priming is resistant to changes in pitch height (within the tested context). This is a reassuring finding and represents an important contribution to the literature on tonality in music cognition. It supports the notion that tonal priming is primarily a cognitive rather than a sensory phenomenon (Bigand et al., 2003). But these experiments also raise questions about the integration of schematic expectations and prompt further research in this area to support the tonal priming literature.

Author note Thanks to Jessica Yap, Clarisse Chen, Booka Nile, and Sonia Ceglinski for collecting some of the data.

\section{References}

Bharucha, J. J. (1984). Anchoring effects in music-The resolution of dissonance. Cognitive Psychology, 16, 485-518.

Bharucha, J. J. (1987). Music cognition and perceptual facilitation-A connectionist framework. Music Perception, 5, 1-30.

Bharucha, J. J., \& Stoeckig, K. (1986). Reaction time and musical expectancy: Priming of chords. Journal of Experimental Psychology: Human Perception and Performance, 12, 403-410. doi:10.1037/ 0096-1523.12.4.403

Bharucha, J. J., \& Stoeckig, K. (1987). Priming of chords: Spreading activation or overlapping frequency spectra? Perception \& Psychophysics, 41, 519-524.

Bigand, E., Delbé, C., Poulin-Charronnat, B., Leman, M., \& Tillmann, B. (2014). Empirical evidence for musical syntax processing? Computer simulations reveal the contribution of auditory shortterm memory. Frontiers in Systems Neuroscience, 8(94), 1-27. doi:10.3389/fnsys.2014.00094

Bigand, E., Poulin, B., Tillmann, B., Madurell, F., \& D'Adamo, D. A. (2003). Sensory versus cognitive components in harmonic priming. Journal of Experimental Psychology: Human Perception and Performance, 29, 159-171. doi:10.1037/0096-1523.29.1.159

Bigand, E., \& Poulin-Charronnat, B. (2006). Are we "experienced listeners"? A review of the musical capacities that do not depend on formal musical training. Cognition, 100, 100-130. doi:10.1016/j. cognition.2005.11.007

Bigand, E., Tillmann, B., Poulin, B., D’Adamo, D. A., \& Madurell, F. (2001). The effect of harmonic context on phoneme monitoring in vocal music. Cognition, 81, B11-B20.

Bigand, E., Tillmann, B., Poulin-Charronnat, B., \& Manderlier, D. (2005). Repetition priming: Is music special? Quarterly Journal of Experimental Psychology, 58A, 1347-1375. doi:10.1080/ 02724980443000601

Boltz, M. G. (1989). Perceiving the end: Effects of tonal relationships on melodic completion. Journal of Experimental Psychology: Human Perception and Performance, 15, 749-761.

Brainard, D. H. (1997). The psychophysics toolbox. Spatial Vision, 10, 433-436. doi:10.1163/156856897X00357
Bregman, A. S. (1990). Auditory scene analysis: The perceptual organization of sound. Cambridge, MA: MIT Press.

Brown, S., \& Jordania, J. (2013). Universals in the world's musics. Psychology of Music, 41, 229-248.

Burns, E. M. (1999). Intervals, scales, and tuning. In D. Deutsch (Ed.), The psychology of music (Vol. 2, pp. 215-264). New York, NY: Academic Press.

Collins, T., Tillmann, B., Barrett, F. S., Delbé, C., \& Janata, P. (2014). A combined model of sensory and cognitive representations underlying tonal expectations in music: From audio signals to behavior. Psychological Review, 121, 33-65. doi:10.1037/a0034695

Cuddy, L. L., Cohen, A. J., \& Mewhort, D. J. K. (1981). Perception of structure in short melodic sequences. Journal of Experimental Psychology: Human Perception and Performance, 7, 869-883. doi:10.1037/0096-1523.7.4.869

Demany, L., \& Armand, F. (1984). The perceptual reality of tone chroma in early infancy. Journal of the Acoustical Society of America, 76, 57-66. doi:10.1121/1.391006

Deutsch, D. (1972). Octave generalization and tune recognition. Perception \& Psychophysics, 11, 411-412.

Deutsch, D. (Ed.). (2013). The psychology of music (3rd ed.). San Diego, CA: Academic Press.

Dowling, W. J. (1973). Perception of interleaved melodies. Cognitive Psychology, 5, 322-337.

Dowling, W. J. (1978). Scale and contour: Two components of a theory of memory for melodies. Psychological Review, 85, 341-354. doi:10. 1037/0033-295X.85.4.341

Dowling, W. J. (1984). Musical experience and tonal scales in the recognition of octave-scrambled melodies. Psychomusicology, 4, 13-32. doi: $10.1037 / \mathrm{h} 0094206$

Dowling, W. J., \& Hollombe, A. W. (1977). The perception of melodies distorted by splitting into several octaves: Effects of increasing proximity and melodic contour. Perception \& Psychophysics, 21, 60-64.

Escoffier, N., \& Tillmann, B. (2008). The tonal function of a taskirrelevant chord modulates speed of visual processing. Cognition, 107, 1070-1083. doi:10.1016/j.cognition.2007.10.007

Hoeschele, M., Weisman, R. G., \& Sturdy, C. B. (2012). Pitch chroma discrimination, generalization, and transfer tests of octave equivalence in humans. Attention, Perception, and Psychophysics, 74, 1742-1760. doi:10.3758/s13414-012-0364-2

Huron, D. (2001). Tone and voice: A derivation of the rules of voiceleading from perceptual principles. Music Perception, 19, 1-64. doi: 10.1525/mp.2001.19.1.1

Huron, D., Kinney, D., \& Precoda, K. (2006). Influence of pitch height on the perception of submissiveness and threat in musical passages. Empirical Musicology Review, 1, 170-177.

Janata, P., Birk, J. L., Van Horn, J. D., Leman, M., Tillmann, B., \& Bharucha, J. J. (2002). The cortical topography of tonal structures underlying Western music. Science, 298, 2167-2170.

Juslin, P. N., \& Laukka, P. (2003). Communication of emotions in vocal expression and music performance: Different channels, same code? Psychological Bulletin, 129, 770-814. doi:10.1037/0033-2909.129. 5.770

Kallman, H. J., \& Massaro, D. W. (1979). Tone chroma is functional in melody recognition. Perception \& Psychophysics, 26, 32-36.

Krumhansl, C. L. (1979). Psychological representation of musical pitch in a tonal context. Cognitive Psychology, 11, 346-374.

Krumhansl, C. L. (1990). Cognitive foundations of musical pitch. New York, NY: Oxford University Press.

Krumhansl, C. L., \& Kessler, E. J. (1982). Tracing the dynamic changes in perceived tonal organization in a spatial representation of musical keys. Psychological Review, 89, 334-368. doi:10.1037/0033-295X. 89.4.334

Krumhansl, C. L., \& Schmuckler, M. A. (1986, July). Key-finding in music: An algorithm based on pattern matching to tonal 
hierarchies. Paper presented at the 19th Annual Meeting of the Society of Mathematical Psychology, Cambridge, MA.

Krumhansl, C. L., \& Shepard, R. N. (1979). Quantification of the hierarchy of tonal functions within a diatonic context. Journal of Experimental Psychology: Human Perception and Performance, 5, 579-594. doi:10.1037/0096-1523.5.4.579

Leman, M. (2000). An auditory model of the role of short-term memory in probe-tone ratings. Music Perception, 17, 481-509.

Marmel, F., Perrin, F., \& Tillmann, B. (2011). Tonal expectations influence early pitch processing. Journal of Cognitive Neuroscience, 23, 3095-3104. doi:10.1162/jocn.2011.21632

Marmel, F., \& Tillmann, B. (2009). Tonal priming beyond tonics. Music Perception, 26, 211-221.

Marmel, F., Tillmann, B., \& Delbe, C. (2010). Priming in melody perception: Tracking down the strength of cognitive expectations. Journal of Experimental Psychology: Human Perception and Performance, 36, 1016-1028. doi:10.1037/a0018735

Marmel, F., Tillmann, B., \& Dowling, W. J. (2008). Tonal expectations influence pitch perception. Perception \& Psychophysics, 70, 841852. doi:10.3758/PP.70.5.841

Mathworks. (2004). Matlab. Natick, MA: The Mathworks, Inc.

Meyer, L. B. (1973). Explaining music: Essays and explorations. Berkeley, CA: University of California Press.

Narmour, E. (1990). The analysis and cognition of basic melodic structures: The implication-realization model. Chicago, IL: University of Chicago Press.

Pashler, H., \& Wagenmakers, E.-J. (2012). Editors' introduction to the special section on replicability in psychological science: A crisis of confidence? Perspectives on Psychological Science, 7, 528-530. doi:10.1177/1745691612465253

Poulin-Charronnat, B., Bigand, E., \& Madurell, F. (2005). The influence of voice leading on harmonic priming. Music Perception, 22, 613627. doi:10.1525/mp.2005.22.4.613

Russo, F. A., Cuddy, L. L., Galembo, A., \& Thompson, W. F. (2007). Sensitivity to tonality across the pitch range. Perception, 36, 781790. doi:10.1068/p5435

Schmuckler, M. A. (2004). Pitch and pitch structures. In J. Neuhoff(Ed.), Ecological psychoacoustics (pp. 271-315). San Diego, CA: Elsevier Science.

Schmuckler, M. A. (2009). Components of melodic processing. In S. Hallam, I. Cross, \& M. Thaut (Eds.), Oxford handbook of music psychology (pp. 93-106). Oxford, UK: Oxford University Press.
Shepard, R. N. (1964). Circularity in judgments of relative pitch. Journal of the Acoustical Society of America, 36, 2346-2353.

Shepard, R. N. (1982). Geometrical approximations to the structure of musical pitch. Psychological Review, 89, 305-333. doi:10.1037/ 0033-295X.89.4.305

Tekman, H. G., \& Bharucha, J. J. (1998). Implicit knowledge versus psychoacoustic similarity in priming of chords. Journal of Experimental Psychology: Human Perception and Performance, 24, 252-260. doi:10.1037/0096-1523.24.1.252

Tillmann, B., Bharucha, J. J., \& Bigand, E. (2000). Implicit learning of tonality: A self-organizing approach. Psychological Review, 107, 885-913. doi:10.1037/0033-295X.107.4.885

Tillmann, B., Janata, P., Birk, J., \& Bharucha, J. J. (2003a). The costs and benefits of tonal centers for chord processing. Journal of Experimental Psychology: Human Perception and Performance, 29, 470-482. doi:10.1037/0096-1523.29.2.470

Tillmann, B., Janata, P., Birk, J., \& Bharucha, J. J. (2008). Tonal centers and expectancy: Facilitation or inhibition of chords at the top of the harmonic hierarchy? Journal of Experimental Psychology: Human Perception and Performance, 34, 1031-1043. doi:10.1037/00961523.34.4.1031

Tillmann, B., Janata, P., \& Bharucha, J. J. (2003b). Activation of the inferior frontal cortex in musical priming. Cognitive Brain Research, 16, 145-161.

Trainor, L. J., \& Trehub, S. E. (1994). Key membership and implied harmony in Western tonal music: Developmental perspectives. Perception \& Psychophysics, 56, 125-132.

van Noorden, L. (1975). Temporal coherence in the perception of tone sequences ( $\mathrm{PhD}$ doctoral dissertation). Technical University Eindhoven, Eindhoven, The Netherlands.

Vuvan, D. T., \& Schmuckler, M. A. (2011). Tonal hierarchy representations in auditory imagery. Memory \& Cognition, 39, 477-490. doi: 10.3758/s13421-010-0032-5

Warren, J. D., Uppenkamp, S., Patterson, R. D., \& Griffiths, T. D. (2003). Separating pitch chroma and pitch height in the human brain. Proceedings of the National Academy of Sciences of the United States of America, 100, 10038-10042. doi:10.1073/pnas. 1730682100

Wright, A. A., Rivera, J. J., Hulse, S. H., Shyan, M., \& Neiworth, J. J. (2000). Music perception and octave generalization in rhesus monkeys. Journal of Experimental Psychology: General, 129, 291-307. 